\title{
Site-specific genetic engineering in cereals - principles and applications
}

Kumlehn J.

Leibniz Institute of Plant Genetics and Crop Plant Research (IPK) Gatersleben, Germany email: kumlehn@ipk-gatersleben.de

Methods of site-directed genome modification offer great opportunities for fundamental plant research and crop improvement. As a precondition for keeping pace with the rapid development of CRISPR-associated (Cas) endonuclease technology, a modular and versatile vector system has been developed that is based upon the use of type IIS restriction enzymes and hence allows for complex molecular cloning procedures in single reactions. Not only can multiple guide RNAs be expressed simultaneously, also newly emerging system components such as Cas derivatives with improved or novel functionality can be readily tested and utilized. The increasing interest in customizable endonuclease technology is also associated with a renaissance of protoplast culture and transfection, which is of great utility for the development of novel methodical approaches as well as for the functional validation of components before it comes to the typically more laborious targeted genetic modification at the whole-plant level. In addition, options to couple site-directed genetic engineering with haploid technology have been implemented, be it for an efficient genetic fixation of modified alleles or for the use of cas $9 / \mathrm{gRNA}$-transgenic haploidy inducer lines for cross-pollination, which has the capability of triggering target site-specific modifications with much reduced genotype dependence. Cas endonuclease technology has been developed and utilized in cereals to elucidate gene functions and to improve plant performance. Recent results of translational research include the establishment of plant resistance to pathogens and the modification of further yield- and quality-determining plant features. However, a major challenge we are still facing is to establish viable techniques enabling us to generate user-preferred DNA modifications with absolute precision. 